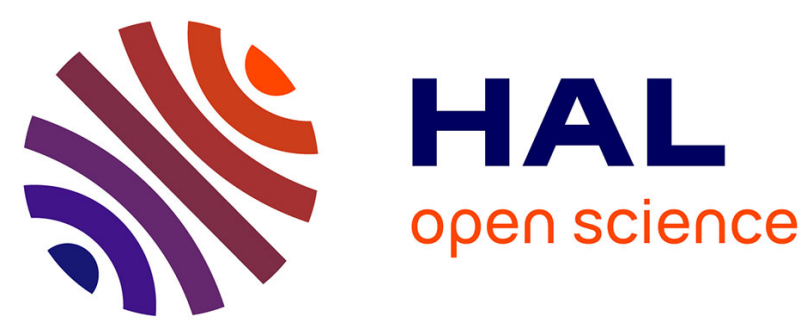

\title{
What frequency bandwidth to run cellular network in a given country? - a downlink dimensioning problem
}

\author{
Bartlomiej Blaszczyszyn, Mohamed Kadhem Karray
}

\section{To cite this version:}

Bartlomiej Blaszczyszyn, Mohamed Kadhem Karray. What frequency bandwidth to run cellular network in a given country? - a downlink dimensioning problem. WiOpt/SpaSWiN, May 2015, Bombay, India. 10.1109/WIOPT.2015.7151119 . hal-01070210v2

\section{HAL Id: hal-01070210 \\ https://hal.inria.fr/hal-01070210v2}

Submitted on 19 Mar 2015

HAL is a multi-disciplinary open access archive for the deposit and dissemination of scientific research documents, whether they are published or not. The documents may come from teaching and research institutions in France or abroad, or from public or private research centers.
L'archive ouverte pluridisciplinaire HAL, est destinée au dépôt et à la diffusion de documents scientifiques de niveau recherche, publiés ou non, émanant des établissements d'enseignement et de recherche français ou étrangers, des laboratoires publics ou privés. 


\title{
What frequency bandwidth to run cellular network in a given country? - a downlink dimensioning problem
}

\author{
Bartłomiej Błaszczyszyn $^{\dagger}$ and Mohamed K. Karray*
}

\begin{abstract}
We propose an analytic approach to the frequency bandwidth dimensioning problem, faced by cellular network operators who deploy/upgrade their networks in various geographical regions (countries) with an inhomogeneous urbanization. We present a model allowing one to capture fundamental relations between users' quality of service parameters (mean downlink throughput), traffic demand, the density of base station deployment, and the available frequency bandwidth. These relations depend on the applied cellular technology (3G or 4G impacting user peak bit-rate) and on the path-loss characteristics observed in different (urban, sub-urban and rural) areas. We observe that if the distance between base stations is kept inversely proportional to the distance coefficient of the path-loss function, then the performance of the typical cells of these different areas is similar when serving the same (per-cell) traffic demand. In this case, the frequency bandwidth dimensioning problem can be solved uniformly across the country applying the mean cell approach proposed in [1]. We validate our approach by comparing the analytical results to measurements in operational networks in various geographical zones of different countries.
\end{abstract}

Index Terms - cellular network; dimensioning; bandwidth; spatial heterogeneity, QoS-homogeneous networks

\section{INTRODUCTION}

A systematic increase of the traffic in wireless cellular networks leads to a potential degradation of the users' quality of service (QoS). In order to prevent such degradation, network operators (besides upgrading the technology) keep adding new base stations and/or supplementary frequency bandwidth. Planning of this network dimensioning process requires the knowledge of the relations between the QoS parameters, traffic demand, density of base station deployment, and the operated frequency bandwidth. Such relations are usually developed for homogeneous network models representing, separately, a typical city or a rural area. To the best of our knowledge, a global approach to some larger geographical zone, as e.g. some given country, typically with an inhomogeneous urbanization, has not yet been proposed. This is the main goal of this paper.

Our work is based on [1], where a typical-cell approach was proposed to the aforementioned study of the QoS, with the information-theoretic characterization of the peak-bit rate, the queueing-theoretic evaluation of the cell performance, and a stochastic-geometric approach to irregular but homogeneous network deployment. In this paper we extend this approach to an inhomogeneous network deployment, i.e., a network which is supposed to cover urban, sub-urban and rural areas, for which path-loss characteristics and the network deployment densities are different.

\footnotetext{
${ }^{\dagger}$ Inria/Ens, 23 av. d’Italie 75214 Paris, France; Bartek.Blaszczyszyn @ens.fr * Orange Labs, 38/40 rue Général Leclerc, 92794 Issy-Moulineaux, France; mohamed.karray@orange.com
}

A key observation that we make in this regard is that if the distance between neighbouring base stations is kept inversely proportional (equivalently, the square root of the network density is proportional) to the distance coefficient of the path-loss function, then the typical cells of these different areas exhibit the same "response" to the traffic demand. Specifically, they exhibit the same relation between the mean user throughput and the per-cell traffic demand. Thus, one can say that this inhomogeneous network offers to its users homogeneous "QoS response" across all different areas. More precisely: cells in rural areas will be larger than these in urban areas, and the traffic demand per surface can be different, with urban areas generally serving more traffic. However, in the case when the mean traffic demand per cell is equal, these areas will "offer" the same user's throughput.

As a consequence, the bandwidth dimensioning problem can be addressed and solved for one particular, say urban, area and applied to the whole network, appropriately adapting the density of base station in sub-urban and rural areas to the specific value of the distance coefficient in the path-loss function.

We validate our approach by comparing the obtained results to measurements in $3 \mathrm{G}$ and $4 \mathrm{G}$ operational networks in different countries in Europe, Africa and Asia.

\section{A. Related works}

The evaluation of the performance of cellular networks is a complex problem, but crucial for network operators. It motivates a lot of engineering and research studies. The complexity of this problem made many actors develop complex and time consuming simulation tools such as those developed by the industrial contributors to 3GPP (3rd Generation Partnership Project) [2]. There are many other simulation tools such as TelematicsLab LTE-Sim [3], University of Vien LTE simulator $[4,5]$ and LENA tool $[6,7]$ of CTTC, which are not necessarily compliant with 3GPP.

A possible analytical approach to this problem relies on the information theoretic characterization of the individual link performance; cf e.g. $[8,9]$, in conjunction with a queueing theoretic modeling and analysis of the user traffic; cf. e.g. [1015]. Recently, $[1,16]$ proposes an approach combining queueing theory and stochastic geometry allowing to study the dependence of the mean user throughput on the traffic demand in a typical city. This analytical approach is validated with field measurements at the scale of a city. At this scale, not only the network average metrics but also their spatial distributions in the considered, homogeneous, zones are well predicted in [17]. This prior work does not consider inhomogeneous networks, observed at the scale of a larger geographical zone 
or a whole country. In particular, the simulation tools are too time consuming to be applicable at this scale.

\section{B. Paper organization}

We describe our general cellular network model, comprising the geometry of base stations, propagation loss model, traffic dynamics, and service policy in Section II. In Section III we propose a model permitting to get the relation of users' QoS to the key network parameters at a country scale. This approach is validated by comparing, in Section IV, the obtained results to measurements in various operational networks in different countries. We show also how to solve the bandwidth dimensioning problem.

\section{HOMOGENEOUS MODEL DESCRIPTION}

In this section we briefly recall the model from [1].

\section{A. Network geometry and propagation}

The network is composed of base stations (BS) whose locations are modelled by a stationary point process $\Phi=\left\{X_{n}\right\}_{n \in \mathbb{Z}}$ on $\mathbb{R}^{2}$ with intensity $\lambda>0$. We assume that $\Phi$ is stationary and ergodic. Each base station $X_{n}$ emits a power denoted by $P_{n}$. We assume that $\left\{P_{n}\right\}_{n \in \mathbb{Z}}$ are i.i.d. marks of $\Phi$.

The propagation loss comprises a deterministic effect depending on the distance between the transmitter and receiver called path-loss, and a random effect called shadowing. The path-loss is modeled by a function

$$
l(x)=(K|x|)^{\beta}, \quad x \in \mathbb{R}^{2},
$$

where $K>0$ and $\beta>2$ are two parameters called distance coefficient and exponent respectively. The shadowing between BS $X_{n}$ and all the locations $y \in \mathbb{R}^{2}$ is modelled by a stochastic process $S_{n}\left(y-X_{n}\right)$. We assume that the processes $\left\{S_{n}(\cdot)\right\}_{n \in \mathbb{Z}}$ are i.i.d. marks of $\Phi$.

The inverse of the power received at location $y$ from BS $X_{n}$ is denoted by

$$
L_{X_{n}}(y)=\frac{l\left(y-X_{n}\right)}{P_{n} S_{n}\left(y-X_{n}\right)}, \quad y \in \mathbb{R}^{2}, n \in \mathbb{Z}
$$

and will be called, with a slight abuse of terminology, propagation loss between $y$ and $X_{n}$.

In order to simplify the notation, we shall omit the index $n$ of BS $X_{n}$. Each BS $X \in \Phi$ serves the locations where the received power is the strongest among all the $\mathrm{BS}$; that is

$$
V(X)=\left\{y \in \mathbb{R}^{2}: L_{X}(y) \leq L_{Y}(y) \text { for all } Y \in \Phi\right\}
$$

called cell of $X$.

The signal-to-interference-and-noise (SINR) power ratio in the downlink for a user located at $y \in V(X)$ equals

$$
\operatorname{SINR}(y, \Phi)=\frac{1 / L_{X}(y)}{N+\sum_{Y \in \Phi \backslash\{X\}} \varphi_{Y} / L_{Y}(y)},
$$

where $N$ is the noise power and each $\varphi_{Y} \in[0,1]$ is some interference factor accounting for the activity of $\mathrm{BS} Y$ in a way that will be made specific in Section II-C. We assume that $\left\{\varphi_{Y}\right\}_{Y \in \Phi}$ are additional (not necessarily independent) marks of the point process $\Phi$.

A single user served by BS $X$ and located at $y \in V(X)$ gets a bit-rate $R(\operatorname{SINR}(y, \Phi))$, called peak bit-rate, which is some function of the SINR given by (3). Particular form of this function depends on the actual technology used to support the wireless link.

\section{B. Traffic dynamics}

We consider variable bit-rate (VBR) traffic; i.e., users arrive to the network and require to transmit some volume of data at a bit-rate decided by the network. Each user arrives at a location uniformly distributed and requires to transmit a random volume of data of mean $1 / \mu$. The duration between the arrivals of two successive users in each zone of surface $S$ is an exponential random variable of parameter $\gamma \times S$ (i.e. there are $\gamma$ arrivals per surface unit). The arrival locations, inter-arrival durations as well as the data volumes are assumed independent of each other. We assume that the users don't move during their calls. The traffic demand per surface unit is then equal to $\rho=\gamma / \mu$, which may be expressed in bit $/ \mathrm{s} / \mathrm{km}^{2}$.

The traffic demand to a given cell equals

$$
\rho(X)=\rho|V(X)|, \quad X \in \Phi,
$$

where $|A|$ is the surface of $A$.

We shall assume that each user in a cell gets an equal portion of time for his service. Thus when there are $k$ users in a cell, each one gets a bit-rate equal to his peak bit-rate divided by $k$. More explicitly, if a base station located at $X$ serves $k$ users located at $y_{1}, y_{2}, \ldots, y_{k} \in V(X)$ then the bit-rate of the user located at $y_{j}$ equals $\frac{1}{k} R\left(\operatorname{SINR}\left(y_{j}, \Phi\right)\right), j \in\{1,2, \ldots, k\}$.

\section{Cell performance metrics}

We consider now the stationary state of the network in the long run of the call arrivals and departures. Using queuing theory tools, it is proven in [15, Proposition 1] that:

- Each base station $X \in \Phi$ can serve the traffic demand within its cell if this latter doesn't exceed some critical value which is the harmonic mean of the peak bit-rate over the cell; that is

$$
\rho_{\mathrm{c}}(X):=|V(X)|\left[\int_{V(X)} 1 / R(\operatorname{SINR}(y, \Phi)) \mathrm{d} y\right]^{-1},
$$

- The mean user throughput in cell $V(X)$ equals

$$
r(X)=\max \left(\rho_{\mathrm{c}}(X)-\rho(X), 0\right) .
$$

- The mean number of users in cell $V(X)$ equals

$$
N(X)=\frac{\rho(X)}{r(X)} \text {. }
$$

- Moreover, we define the cell load as

$$
\theta(X)=\frac{\rho(X)}{\rho_{\mathrm{c}}(X)}=\rho \int_{V(X)} 1 / R(\operatorname{SINR}(y, \Phi)) \mathrm{d} y .
$$

- The probability that the base station has at least one user to serve (at a given time) equals

$$
p(X)=\min (\theta(X), 1) \text {. }
$$


We assume that a BS transmits only when it serves at least one user. Then, as proposed in [1], we take $\varphi_{Y}=p(Y)$ in the SINR expression (3). Thus (8) becomes

$$
\theta(X)=\rho \int_{V(X)} 1 / R\left(\frac{1 / L_{X}(y)}{N+\sum_{Y \in \Phi \backslash\{X\}} \frac{\min (\theta(Y), 1)}{L_{Y}(y)}}\right) \mathrm{d} y,
$$

which is a system of equations with unknown cell loads $\{\theta(X)\}_{X \in \Phi}$.

\section{Network performance metrics}

The network performance metrics are defined by averaging spatially over all the cells in the network in an appropriate way [1]. In particular, it follows from the ergodic theorem for point processes [18, Theorem 13.4.III] that the average cell load equals

$$
\lim _{|A| \rightarrow \infty} \frac{\sum_{X \in \Phi \cap A} \theta(X)}{\Phi(A)}=\mathbf{E}^{0}[\theta(0)]=\frac{\rho}{\lambda} \mathbf{E}\left[\frac{1}{R(\operatorname{SINR}(0, \Phi))}\right]
$$

where $A$ is a ball centered at the origin having radius increasing to infinity and $\mathbf{E}^{0}$ is the expectation with respect to the Palm probability associated to $\Phi$. The second equality in the above equation is proved in [1, Proposition 3].

Moreover, it is observed in [1] that the network performance metrics are well approximated by the mean cell model. Specifically, we define a virtual cell having traffic demand $\bar{\rho}:=\mathbf{E}^{0}[\rho(0)]$ and load $\bar{\theta}:=\mathbf{E}^{0}[\theta(0)]$. For other performance metrics, the mean cell mimics the behavior of the true cells given in Section II-C; that is it has critical traffic demand deduced from (8)

$$
\bar{\rho}_{\mathrm{c}}:=\frac{\bar{\rho}}{\bar{\theta}},
$$

mean user's throughput deduced from (6)

$$
\bar{r}:=\max \left(\bar{\rho}_{\mathrm{c}}-\bar{\rho}, 0\right),
$$

and the mean number of users deduced from (7)

$$
\bar{N}:=\frac{\bar{\rho}}{\bar{r}} \text {. }
$$

The load equations (10) become for the mean cell

$$
\bar{\theta}=\frac{\rho}{\lambda_{\mathrm{BS}}} \mathbf{E}\left[1 / R\left(\frac{1 / L_{X^{*}}(0)}{N+\bar{\theta} \sum_{Y \in \Phi \backslash\{X\}} 1 / L_{Y}(0)}\right)\right],
$$

where $X^{*}$ is the location of the BS whose cell covers the origin.

\section{FROM SCALING EQUATIONS TO INHOMOGENEOUS NETWORKS}

We begin by studying some scaling laws observed in homogeneous networks. Then we will introduce some inhomogeneous networks which are able to offer homogeneous QoS response to the traffic demand.

\section{A. Scaling laws for homogeneous networks}

Consider a homogeneous network model descried in Section II. For $\alpha>0$ consider a network obtained from this original one by scaling the base station locations $\Phi^{\prime}=$ $\left\{X^{\prime}=\alpha X\right\}_{X \in \Phi}$, the traffic demand intensity $\rho^{\prime}=\rho / \alpha^{2}$, distance coefficient $K^{\prime}=K / \alpha$ and shadowing processes $S_{n}^{\prime}(y)=S_{n}\left(\frac{y}{\alpha}\right)$, while preserving the original (arbitrary) marks (interference factors) $\varphi_{X^{\prime}}^{\prime}=\varphi_{X}, X \in \Phi$ and powers $P_{n}^{\prime}=P_{n}$. For the rescaled network consider the cells $V^{\prime}\left(X^{\prime}\right)$ given by (2) and their characteristics $\rho^{\prime}\left(X^{\prime}\right), \rho_{\mathrm{c}}^{\prime}\left(X^{\prime}\right), r^{\prime}\left(X^{\prime}\right)$, $N^{\prime}\left(X^{\prime}\right), \theta^{\prime}\left(X^{\prime}\right), p^{\prime}\left(X^{\prime}\right)$ calculated as in (4), (5), (6), (7), (8) and (9), respectively.

Proposition 1: For any $X^{\prime} \in \Phi^{\prime}$, we have $V^{\prime}\left(\alpha X_{n}\right)=$ $\alpha V\left(X_{n}\right)$ while $\rho^{\prime}\left(X^{\prime}\right)=\rho(X), \rho_{\mathrm{c}}^{\prime}\left(X^{\prime}\right)=\rho_{\mathrm{c}}(X), r^{\prime}\left(X^{\prime}\right)=$ $r(X), N^{\prime}\left(X^{\prime}\right)=N(X), \theta^{\prime}\left(X^{\prime}\right)=\theta(X)$.

Proof. Observe that

$$
L_{\alpha X_{n}}^{\prime}(y)=\frac{P_{n} S_{n}^{\prime}\left(y-\alpha X_{n}\right)}{\left(K^{\prime}\left|y-\alpha X_{n}\right|\right)^{\beta}}=\frac{P_{n} S_{n}\left(\frac{y}{\alpha}-X_{n}\right)}{\left(K\left|\frac{y}{\alpha}-X_{n}\right|\right)^{\beta}}=L_{X_{n}}\left(\frac{y}{\alpha}\right)
$$

and by (2) one gets the required dilation relation for the individual cells. Moreover, using (3) one gets by simple algebra $\operatorname{SINR}\left(y, \Phi^{\prime}\right)=\operatorname{SINR}(y / \alpha, \Phi)$. Starting from (5) and making the change of variable $z=y / \alpha$, it follows that

$$
\begin{aligned}
\rho_{\mathrm{c}}^{\prime}\left(X^{\prime}\right) & =\left|V^{\prime}\left(X^{\prime}\right)\right|\left[\int_{V^{\prime}\left(X^{\prime}\right)} 1 / R\left(\operatorname{SINR}\left(y, \Phi^{\prime}\right)\right) \mathrm{d} y\right]^{-1} \\
& =\alpha^{2}|V(X)|\left[\int_{\alpha V(X)} 1 / R(\operatorname{SINR}(y / \alpha, \Phi)) \mathrm{d} y\right]^{-1} \\
& =\rho_{\mathrm{c}}(X) .
\end{aligned}
$$

The remaining desired equalities then follow from the fact that

$$
\rho^{\prime}\left(X^{\prime}\right)=\frac{\rho}{\alpha^{2}}|\alpha V(X)|=\rho|V(X)|=\rho(X) .
$$

Corollary 1: Consider the rescaled network as in Proposition 1 with the interference factors taken $\varphi_{X}=$ $\min (\theta(X), 1)$. Then the load equations (10) are the same for the two networks $\Phi$ and $\Phi^{\prime}$. Therefore, the load factors solving these equation are the same $\theta^{\prime}\left(X^{\prime}\right)=\theta(X)$ and consequently, $\rho_{\mathrm{c}}^{\prime}\left(X^{\prime}\right)=\rho_{\mathrm{c}}(X), r^{\prime}\left(X^{\prime}\right)=r(X), N^{\prime}\left(X^{\prime}\right)=N(X)$, $\theta^{\prime}\left(X^{\prime}\right)=\theta(X)$.

From the above observations we can deduce now that the considered scaling of the network parameters preserves the mean cell characteristics defined in Section II-D. Denote by $\mathbf{E}^{\prime 0}$ the expectation with respect to the Palm probability associated to $\Phi^{\prime}$.

Corollary 2: Consider the rescaled network as in Proposition 1 with arbitrary interference factors, possibly satisfying the load equations (10). Then $\mathbf{E}^{\prime 0}\left[\rho^{\prime}(0)\right]=\mathbf{E}^{0}[\rho(0)]$ and $\mathbf{E}^{\prime 0}\left[\theta^{\prime}(0)\right]=\mathbf{E}^{0}[\theta(0)]$. Consequently, the mean cells characteristics associated to $\Phi$ and $\Phi^{\prime}$ as described in Section II-D are identical.

Proof. It follows from the inverse formula of Palm calculus [19, Theorem 4.2.1] that $\mathbf{E}^{0}[\rho(0)]=\frac{\rho}{\lambda}$. Similarly, $\mathbf{E}^{\prime 0}\left[\rho^{\prime}(0)\right]=\frac{\rho^{\prime}}{\lambda^{\prime}}=\frac{\rho}{\lambda}$ which proves the first desired equality. 
The second equality follows e.g. by the ergodic argument

$$
\begin{aligned}
\mathbf{E}^{\prime 0}\left[\theta^{\prime}(0)\right] & =\lim _{|A| \rightarrow \infty} \frac{1}{\Phi^{\prime}(A)} \sum_{X^{\prime} \in \Phi^{\prime} \cap A} \theta^{\prime}\left(X^{\prime}\right) \\
& =\lim _{|A| \rightarrow \infty} \frac{1}{\Phi(A / \alpha)} \sum_{X \in \Phi \cap A / \alpha} \theta(X) \\
& =\lim _{|A| \rightarrow \infty} \frac{1}{\Phi(A)} \sum_{X \in \Phi \cap A} \theta(X)=\mathbf{E}^{0}[\theta(0)] .
\end{aligned}
$$

where the second equality is due to Proposition 1.

\section{$B$. Inhomogeneous networks with homogeneous QoS response to the traffic demand}

Consider a geographic region (say a country), which is composed of urban, suburban and rural areas. The parameters $K$ and $\beta$ of the distance loss model (1) depend on the type of the zone. For example the COST-Hata model [20] gives the distance loss function in the form $10 \log _{10}(l(x))=$ $A+B \log _{10}(|x|)$ where $A$ and $B$ are given in Table I and the distance $|x|$ is in $\mathrm{km}$. The corresponding value of the parameter $K=10^{A / B}$ as well as the ratio $K_{\text {urban }} / K$ are also given in this table.

The density of base stations also depends on the type of the zone: it is usually much higher in urban than in rural areas. Indeed, the distance $D$ between two neighboring base stations in rural areas may be up to 10 times larger than in dense urbane zones (where a typical value of $D$ is about $1 \mathrm{~km}$ ).

Consider now a situation where the average distance between neighbouring base stations is kept inversely proportional to the distance coefficient of the path-loss function: $D \times K=$ const, or, in other words,

$$
K_{i} / \sqrt{\lambda}_{i}=\text { const, }
$$

where $\lambda_{i}$ is the network density in the given zone (assumed homogeneous) and $K_{i}$ is the path-loss distance factor observed in this zone. In our example shown in Table I we should thus have $D=1,5$ and $8 \mathrm{~km}$ respectively for urban, suburban and rural zones. 1

Then the scaling laws proved in Section III-A say that locally, for each homogeneous area of this inhomogeneous network, one will observe the same relation between the mean user throughput and the (per-cell) traffic demand. Cells in rural areas will be larger than these in urban areas, however in the case when they are charged in the same way by the traffic demand (which, per unit surface, needs to be correspondingly larger in urban areas) then they offer the same QoS. In other words, one relation is enough to capture the key dependence between the QoS, network density, bandwidth and the traffic demand for different areas of this network. It can be used for the network dimensioning. ${ }^{2}$

\footnotetext{
${ }^{1}$ Indeed, when operators deploys networks, they firstly aim to assure some coverage condition which has the form $(D \times K)^{\beta}=$ const. Moreover, in urban zones, networks have to be densified not only for capacity constraints, but also to assure coverage for indoor users.

${ }^{2}$ The approximation of an inhomogeneous network by a piecewise homogeneous network can be made more precise e.g. following the ideas presented in $[21,22]$. Note also that in our approach we ignored the fact that the pathloss exponent $\beta$ is not the same for urban and the two other zones considered in Table I. This is clearly an approximation. Its quality can be studied using a network equivalence approach [23] allowing for different path-loss exponents.
}

\begin{tabular}{|l|l|l|l|l|}
\hline Environment & $A$ & $B$ & $K=10^{A / B}$ & $K_{\text {urban }} / K$ \\
\hline Urban & 133.1 & 33.8 & 8667 & 1 \\
\hline Suburban & 102.0 & 31.8 & 1612 & 5 \\
\hline Rural & 97.0 & 31.8 & 1123 & 8 \\
\hline
\end{tabular}

PROPAGATION PARAMETERS FOR CARRIER FREQUENCY $1795 \mathrm{MHZ}$ FROM [24, TABLE 6.4].

\section{NUMERICAL RESUltS}

The relations of the network performance metrics to the traffic demand described in Section II-D were already validated in [1] comparing them to operational network measurements in some typical cities in Europe. Thus we shall focus on the validation of its extension to more large areas, and ultimately to a whole country as proposed in Section III. Once this validation carried, we shall solve numerically the bandwidth dimensioning problem.

\section{A. Real-field measurements}

We describe now the real-field measurements. The raw data are collected using a specialized tool which is used by operational engineers for network maintenance. This tool measures several parameters for every base station 24 hours a day. In particular, one can get the traffic demand, cell load and number of users for each cell in each hour. Then we estimate the network performance metrics for each hour averaging over all considered cells. The mean user throughput is calculated as the ratio of the mean traffic demand to the mean number of users. The mean traffic demand is used as the input of our analytical model.

\section{B. A reference country}

We consider a reference country, called in what follows Country 1 , divided into 5 regions. Each of these regions comprises a large mix of urban, suburban and rural zones. In what follows we consider $3 \mathrm{G}$ and $4 \mathrm{G}$ networks deployed in Country 1.

\section{1) $3 G$ network:}

a) Numerical setting: Country 1 is covered by a $3 \mathrm{G}$ network at carrier frequency $f_{0}=2.1 \mathrm{GHz}$ with frequency bandwidth $W=5 \mathrm{MHz}$, base station power $P=60 \mathrm{dBm}$ and noise power $N=-96 \mathrm{dBm}$. The peak bit-rate equals to $30 \%$ of the ergodic capacity of the flat fading channel; i.e., $R(\mathrm{SINR})=0.3 \times W \mathbf{E}\left[\log _{2}\left(1+|H|^{2} \mathrm{SINR}\right)\right]$ where $|H|^{2}$ is a unit mean exponential random variable representing the fading and $\mathbf{E}[\cdot]$ is the expectation with respect to $H$.

b) Typical urban zone: We consider a typical urban zone (a city) in the considered Country 1. Knowing all BS coordinates and the surface of the deployment zone we deduce the BS density $\lambda=1.15$ stations per $\mathrm{km}^{2}$ which corresponds to an average distance between two neighboring BS of $D_{\mathrm{u}} \simeq 1 / \sqrt{\lambda} \simeq 1 \mathrm{~km}$.

For the analytical model, the locations of BS is modelled by a homogeneous Poisson point process with intensity $\lambda$. The parameters of the distance loss model (1) are estimated from the COST Walfisch-Ikegami model [20] which gives

$$
\beta=3.8, \quad K_{0}=7117 \mathrm{~km}^{-1} .
$$




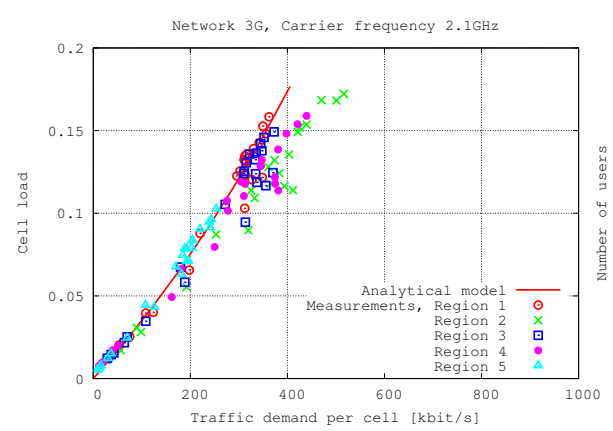

(a) Mean cell load

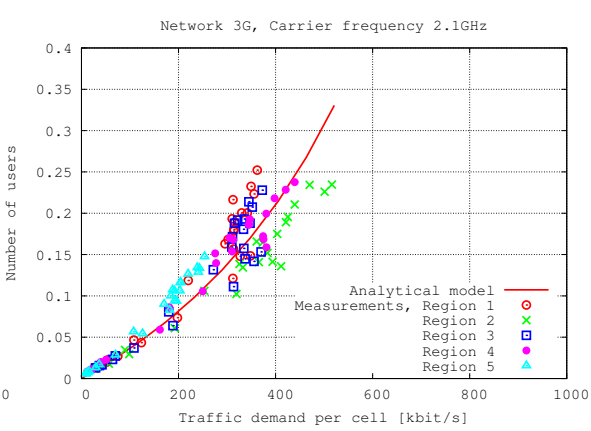

(b) Mean number of users

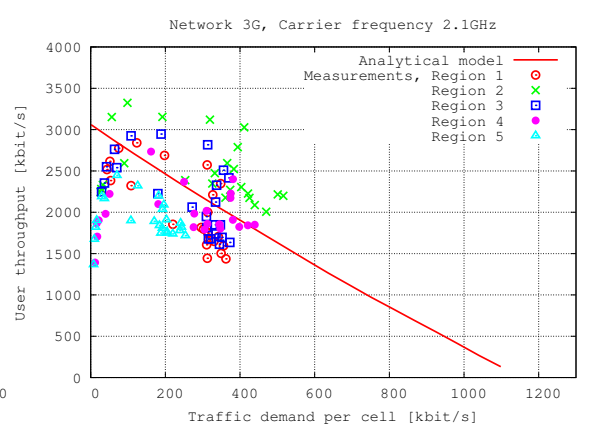

(c) Mean user throughput

Fig. 1. Mean cell characteristics versus traffic demand calculated analytically and estimated from measurements for a 3G network in Country 1.

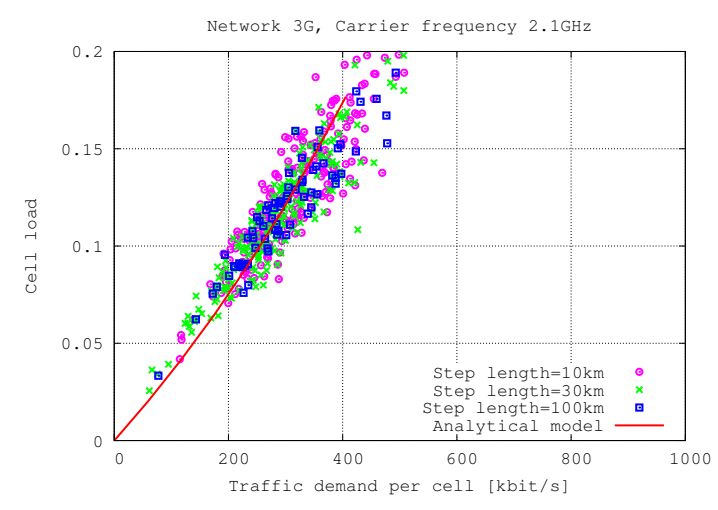

(a) Regular decomposition into squares of different size

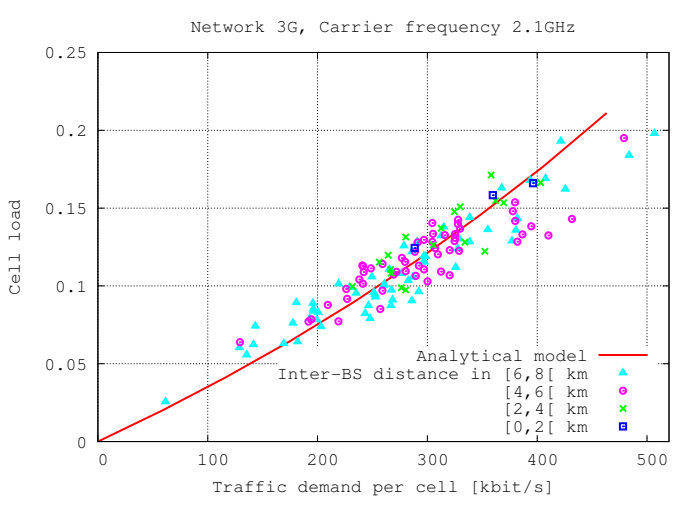

(b) Decomposition into different density zones

Fig. 2. Mean cell load versus traffic demand in a $3 \mathrm{G}$ network at $2.1 \mathrm{GHz}$ in Country 1 , with two different decompositions.

The shadowing random variable is lognormal with unit mean and logarithmic-standard deviation $\sigma=9.6 \mathrm{~dB}$ and mean spatial correlation distance $50 \mathrm{~m}$.

Each BS comprises three antennas having each a threedimensional radiation pattern specified in [2, Table A.2.1.12]. The BS and mobile antenna heights equal $30 \mathrm{~m}$ and $1.5 \mathrm{~m}$ respectively. The pilot channel power is taken equal to $10 \%$ of the total base station power.

The theoretical relations of the network performance metrics to the traffic demand are calculated for such typical urban zone and compared, to measurements in large areas, and ultimately the whole country, applying the approach developed Section III.

c) Network performance: Figure 1(a) shows the mean cell load $\bar{\theta}$ versus mean traffic demand per cell $\bar{\rho}=\rho / \lambda$ calculated analytically and obtained from measurements for the 5 considered regions. The mean number of users $\bar{N}$ and user's throughput $\bar{r}$ versus mean traffic demand per cell $\bar{\rho}$ are plotted in Figures 1(b) and 1(c) respectively. Observe that the analytical curves fits well to the measurements.

In order to analyze the effect of the size of the regions over which we average the cell performance metrics, we decompose Country 1 into a regular grid composed of squares (meshes) of equal side. Figure 2(a) shows the mean cell load versus traffic demand for mesh sizes $3,10,30$ and $100 \mathrm{~km}$ respectively. Observe that the larger is the mesh size, the closer are measurements to the analytical (mean) model.

We aim now to check the hypothesis made in Section III-B, namely that zones with different density of base stations observe the same dependence of the mean cell characteristics on the mean per-cell traffic demand. Figure 2(b) shows the mean cell load versus traffic demand for mesh size $30 \mathrm{~km}$ where we distinguish the meshes according to the distance between neighboring $\mathrm{BS}$; distances within $[0,2] \mathrm{km}$ (resp. $[6,8] \mathrm{km}$ ) corresonding to urban (resp. rural) zones. Observe that there is no apparent difference between urban, sub-urban and rural meshes.

2) $4 G$ network: The peak bit-rate is calculated by $R(\mathrm{SINR})=b \times W \log _{2}(1+\mathrm{SINR} / a)$ where $a=3, b=1.12$ are estimated from fitting to the results of a link simulation tool.

a) Numerical setting for carrier frequency $2.6 \mathrm{GHz}$ : We consider a 4G network at carrier frequency $f=2.6 \mathrm{GHz}$ with frequency bandwidth $W=20 \mathrm{MHz}$, base station power $P=$ $63 \mathrm{dBm}$ and noise power $N=-90 \mathrm{dBm}$.

The typical urban zone is the same as described in Section IV-B1b except for the distance loss parameter $K$. Following the COST-Hata model [20] this latter is calculated by assuming that it is proportional to $f^{2 / \beta}$; which gives $K=K_{0} \times\left(f / f_{0}\right)^{2 / \beta}=7964 \mathrm{~km}^{-1}$.

b) Numerical setting for carrier frequency $800 \mathrm{MHz}$ : We consider also a $4 \mathrm{G}$ network at carrier frequency $f=800 \mathrm{MHz}$ with frequency bandwidth $W=10 \mathrm{MHz}$, base station power $P=60 \mathrm{dBm}$ and noise power $N=-93 \mathrm{dBm}$.

The typical urban zone is the same as described in Section IV-B1b except for the average distance between two neighboring BS which is now $D_{\mathrm{u}} \simeq 1.5 \mathrm{~km}$ and the distance loss parameter $K=K_{0} \times\left(f / f_{0}\right)^{2 / \beta}=4283 \mathrm{~km}^{-1}$. 


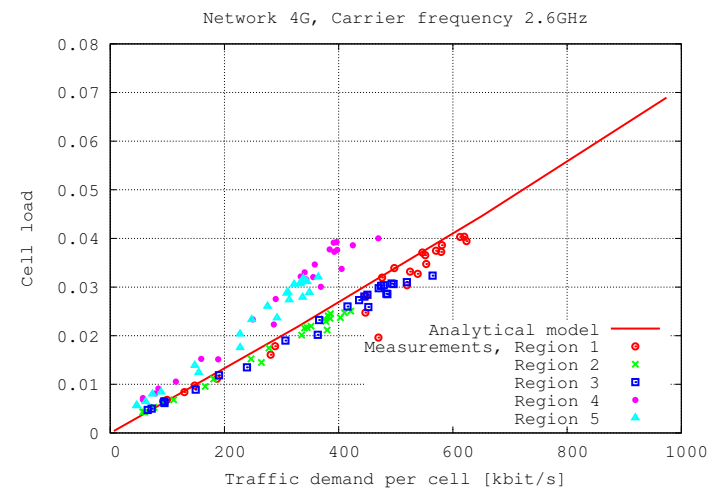

(a) $2.6 \mathrm{GHz}$

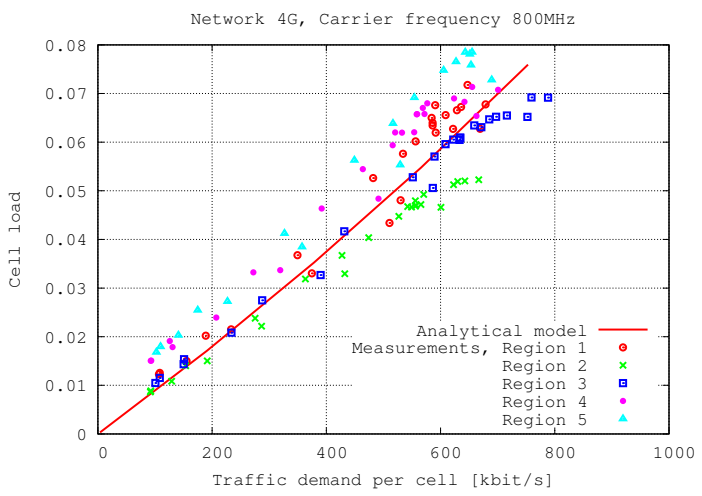

(b) $800 \mathrm{MHz}$

Fig. 3. Mean cell load versus traffic demand for $4 \mathrm{G}$ in Country 1 at two different frequency bandwidths.

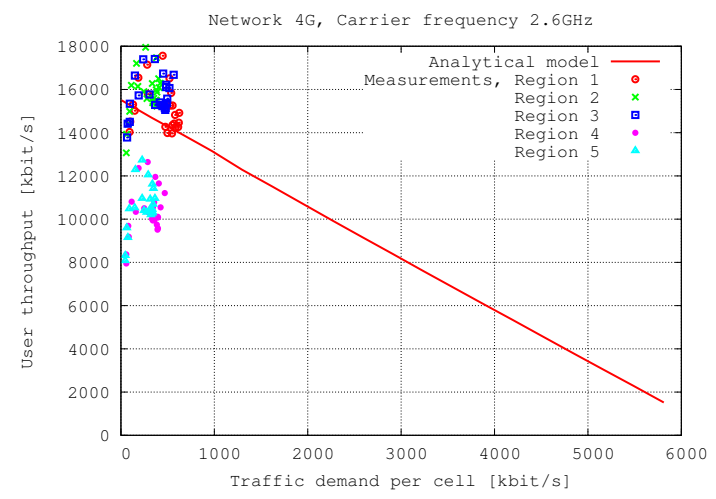

(a) $2.6 \mathrm{GHz}$

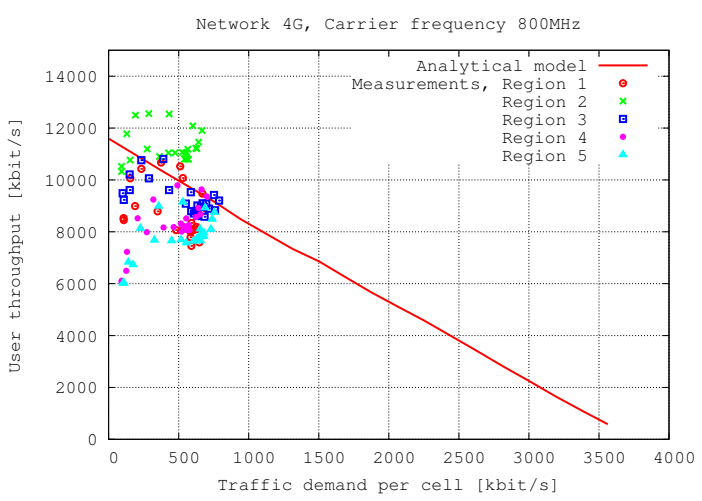

(b) $800 \mathrm{MHz}$

Fig. 4. Mean user throughput versus traffic demand for $4 \mathrm{G}$ in Country 1 at two different frequency bandwidths.

c) Network performance: Figures 3(a) and 3(b) show the mean cell load versus mean traffic demand per cell calculated analytically and obtained from measurements for the $4 \mathrm{G}$ network at carrier frequencies $2.6 \mathrm{GHz}$ and $800 \mathrm{MHz}$ respectively. Observe again that the analytical curve fits well with the measurements.

Figures 4(a) and 4(b) show the mean user's throughput versus mean traffic demand per cell for the $4 \mathrm{G}$ network at carrier frequencies $2.6 \mathrm{GHz}$ and $800 \mathrm{MHz}$ respectively. Even if currently we do not observe traffic demand larger than $1000 \mathrm{kbit} / \mathrm{s}$ per cell, we show the predicted curves in their whole domains as they will serve for the network dimensioning, cf. Section IV-D.

\section{Two other countries}

We consider now two $3 \mathrm{G}$ networks in two other countries, called Country 2 and Country 3 . The numerical setting is the same as in Section IV-B1a. Since we don't have detailed information on the propagation characteristics in these countries, we make the hypothesis that the typical urban zone has the same characteristics as for Country 1; i.e. those described in Section IV-B1b.

Figures 5(a), 5(b) and 5(c) show the mean cell load, users number and user's throughput versus mean traffic demand per cell for the two considered countries. Note that for Country 3 the user's throughput stagnates for traffic demand exceeding $800 \mathrm{kbit} / \mathrm{s} / \mathrm{cell}$. This is due to congestion; some users being blocked at access as may be seen in Figure 5(b).

\section{Bandwidth dimensioning}

We come back now to the question stated in the title of this article: What frequency bandwidth is required to run cellular network guaranteeing sufficient QoS for its users? This is the frequency dimension problem often faced by the network operators.

Here we consider the mean user throughput in the downlink as the QoS metric. We have remarked at the end of Section III-B that (conjecturing) the relation (11) allows one to capture the key dependence between the mean user throughput and the per cell traffic demand (given the network density and the frequency bandwidth) for an inhomogeneous network focusing only on one homogeneous type of network area. We use for this purpose the urban area. Examples of the corresponding user-throughput versus traffic demand curves have been presented on Figures 1(c), 4(a) and 4(b). Note that these curves depend on (increase with) the frequency bandwidth. The solution of the frequency dimensioning problem (for a given technology and a reference, urban, inter-BS distance) consists in finding, for each value of the traffic demand per cell, the minimal frequency bandwidth such that the predicted mean user throughput reaches a given target value.

Figure 6 plots the solutions for this problem in several considered cases. More precisely, it gives the predicted frequency bandwidth allowing one to assure the target $\bar{r}=5 \mathrm{Mbit} / \mathrm{s}$ mean user's throughput in the network, for a given (per cell) traffic demand. We show solutions for $3 \mathrm{G}$ and $4 \mathrm{G}$ networks with 


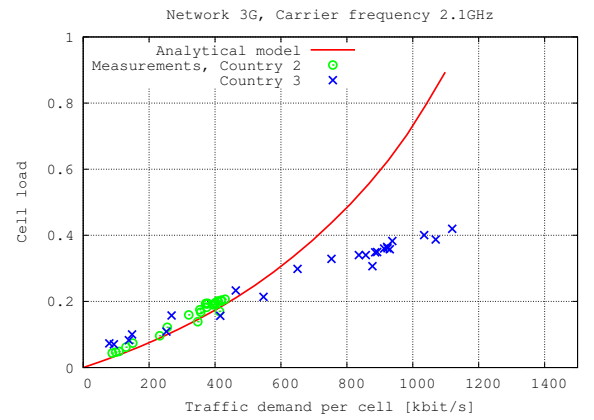

(a) Mean cell load

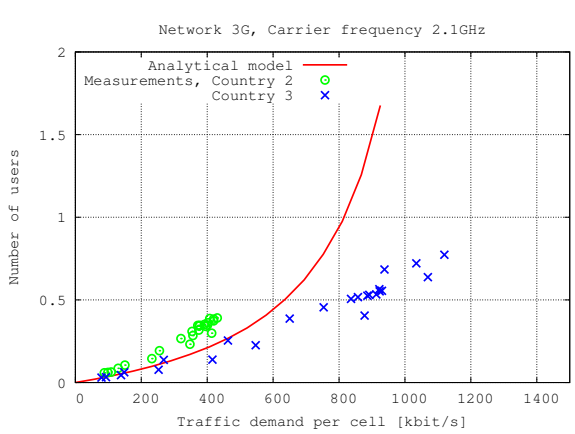

(b) Mean number of users

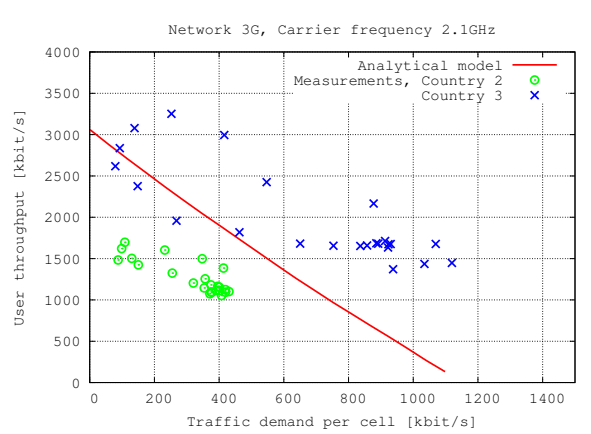

(c) Mean user throughput

Fig. 5. Mean cell characteristics versus traffic demand calculated analytically and estimated from measurements for a $3 \mathrm{G}$ network in Country 2 and 3.

different frequency carriers $f$ and different inter-BS distances $D_{\mathrm{u}}$ relative to the urban area.

As a possible application of the above dimensioning strategy let us mention the following actual problem. In Country 1, the configurations currently deployed for $4 \mathrm{G}$ are $f=2.6 \mathrm{GHz}$ with $D_{\mathrm{u}}=1 \mathrm{~km}$ and $f=800 \mathrm{MHz}$ with $D_{\mathrm{u}}=1.5 \mathrm{~km}$. The operator is wondering whether to densify the network for $f=800 \mathrm{MHz}$ decreasing $D_{\mathrm{u}}$ to $1 \mathrm{~km}$. Figure 6 shows how much bandwidth can be economized in this case.

\section{CONCLUSION}

We propose a model permitting to calculate the performance of $3 \mathrm{G}$ and $4 \mathrm{G}$ wireless cellular networks at the scale of a whole country comprising urban, suburban and rural zones. This model relies on an observation that the distance coefficient in the propagation loss function depends on the type of zone in such a way that its product to the distance between neighboring base stations remains approximately constant. It is then shown that the network performance, in terms of the relations of the mean cell load, number of users and user's throughput to mean traffic demand, are the same for the different types of zones.

This theoretical result is validated by field measurements in $3 \mathrm{G}$ and $4 \mathrm{G}$ cellular networks in various countries. Then we solve the bandwidth dimensioning problem for $3 \mathrm{G}$ and $4 \mathrm{G}$ networks at different carrier frequencies; i.e. we plot the frequency bandwidth as function of the traffic demand per cell to assure a user's throughput of $5 \mathrm{Mbit} / \mathrm{s}$. This curve is crucial for operators to predict the frequency bandwidth required to serve the continuing increase of traffic in the next decades.

We shall attempt in future work to extend the present approach to the uplink; in particular to account for power control effect and the specificity of the locations of the interferers in this case.

Acknowledgement 1: We thank our colleagues from Orange for fruitful exchanges related to the present work.

\section{REFERENCES}

[1] B. Błaszczyszyn, M. Jovanovic, and M. K. Karray, "How user throughput depends on the traffic demand in large cellular networks," in Proc. of WiOpt/SpaSWiN, 2014.

[2] 3GPP, "TR 36.814-V900 Further advancements for E-UTRA Physical Layer Aspects," in 3GPP Ftp Server, 2010.

[3] G. Piro, L. A. Grieco, G. Boggia, F. Capozzi, and P. Camarda, "Simulating LTE cellular systems: an open source framework," IEEE Trans. Veh. Technol., vol. 60, no. 2, 2011.

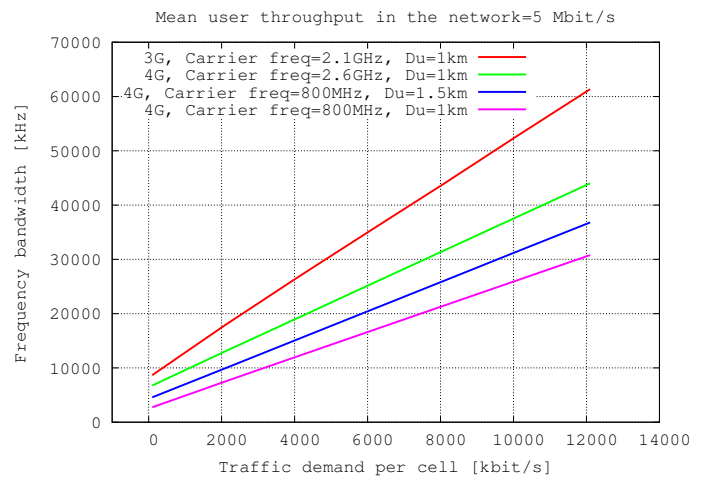

Fig. 6. Bandwidth versus traffic demand per cell to assure a mean user's throughput in the network $\bar{r}=5 \mathrm{Mbit} / \mathrm{s}$.

[4] C. Mehlführer, J. C. Ikuno, M. Simko, S. Schwarz, M. Wrulich, and M. Rupp, "The Vienna LTE simulators - Enabling reproducibility in wireless communications research," EURASIP Journal on Advances in Signal Processing, 2011.

[5] M. Simko, Q. Wang, and M. Rupp, "Optimal pilot symbol power allocation under time-variant channels," EURASIP Journal on Wireless Communications and Networking, no. 25, 2012.

[6] N. Baldo, M. Miozzo, M. Requena, and J. N. Guerrero, "An open source product-oriented LTE network simulator based on ns-3," in Proc. of MSWIM, 2011.

[7] N. Baldo, M. Requena, J. Nin, and M. Miozzo, "A new model for the simulation of the LTE-EPC data plane," in Proc. of WNS3, 2012.

[8] A. J. Goldsmith and S.-G. Chua, "Variable-rate variable-power MQAM for fading channels," IEEE Trans. Commun., vol. 45, pp. 1218-1230, 1997.

[9] P. E. Mogensen, W. Na, I. Z. Kovács, F. Frederiksen, A. Pokhariyal, K. I. Pedersen, T. E. Kolding, K. Hugl, and M. Kuusela, "LTE Capacity Compared to the Shannon Bound," in Proc. of VTC Spring, 2007, pp. 1234-1238.

[10] S. Borst, "User-level performance of channel-aware scheduling algorithms in wireless data networks," in Proc. of IEEE Infocom, 2003

[11] T. Bonald and A. Proutière, "Wireless downlink data channels: user performance and cell dimensioning," in Proc. of Mobicom, Sep. 2003.

[12] N. Hegde and E. Altman, "Capacity of multiservice WCDMA Networks with variable GoS," in Proc. of IEEE WCNC, 2003.

[13] T. Bonald, S. C. Borst, N. Hegde, M. Jonckheere, and A. Proutière, "Flow-level performance and capacity of wireless networks with user mobility," Queueing Systems, vol. 63, no. 1-4, pp. 131-164, 2009.

[14] L. Rong, S. E. Elayoubi, and O. B. Haddada, "Performance evaluation of cellular networks offering TV services," IEEE 
Trans. Veh. Technol., vol. 60, no. 2, pp. 644 -655, feb. 2011.

[15] M. K. Karray and M. Jovanovic, "A queueing theoretic approach to the dimensioning of wireless cellular networks serving variable bit-rate calls," IEEE Trans. Veh. Technol., vol. 62, no. 6, July 2013.

[16] B. Błaszczyszyn, M. Jovanović, and M. K. Karray, "Performance laws of large heterogeneous cellular networks," in In proc. of WiOpt/SpaSWiN, 2015, to appear, see also arxiv: 1411.7785 .

[17] M. Jovanovic, M. K. Karray, and B. Błaszczyszyn, "QoS and network performance estimation in heterogeneous cellular networks validated by real-field measurements," in Proc. of PM2HW2N, 2014.

[18] D. J. Daley and D. VereJones, An introduction to the theory of point processes, 2nd ed. New York: Springer, 2003.

[19] F. Baccelli and B. Błaszczyszyn, Stochastic Geometry and Wireless Networks, Volume I - Theory, ser. Foundations and Trends in Networking. NoW Publishers, 2009, vol. 3, No 3-4.

[20] COST 231, Evolution of land mobile radio (including personal) communications, Final report, Information, Technologies and Sciences, European Commission, 1999.

[21] B. Błaszczyszyn and R. Schott, "Approximate decomposition of some modulated-Poisson Voronoi tessellations," Adv. in Appl. Probab., vol. 35, pp. 847-862, 2003.

[22] B. Błaszczyszyn and R. Schott, "Approximations of functionals of some modulated-Poisson Voronoi tessellations with applications to modeling of communication networks," JJIAM, Special Issue on Voronoi diagrams in Science and Engineering, vol. 22, 2005.

[23] B. Błaszczyszyn and H. P. Keeler, "Equivalence and comparison of heterogeneous cellular networks," in Proc. of PIMRC/WDNCN, 2013.

[24] X. lagrange and P. G. ans S. tabbane, Réseaux GSM-DCS, 2nd ed., Hermes, Ed., 1995. 